REVISTA ACADÉMICA ECO (2020) 22: 1-13

\title{
NEUROCIENCIAS DEL CONSUMIDOR: UNA VISIÓN ACADÉMICA DE SU RECIENTE EVOLUCIÓN
}

Artículo de revisión

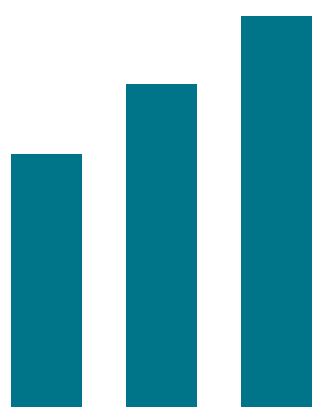

\section{Mario Rolando Paredes Escobar}

Universidad del Rosario, Escuela de Administración, Bogotá, Colombia. Profesor principal de carrera. Doctor en Dirección Empresarial.

Correo: marior.paredes@urosario.edu.com

Fecha de recepción: 10 de noviembre de 2019

Fecha de aceptación: 25 de noviembre de 2019

\section{Resumen}

Este artículo analiza la reciente evolución de la aplicación teórico-práctica de las neurociencias del consumidor. Al especificar algunos hitos de la disciplina como la apropiación del término «neuromarketing», las primeras publicaciones en journals académicos, hasta la creciente proliferación del uso de neurociencias para resolver problemas de marketing en el ámbito comercial y académico, este trabajo intenta responder las siguientes preguntas: ¿cuál ha sido la evolución y el impacto de las neurociencias en el marketing?,¿cuáles son los principales instrumentos que pueden utilizarse para la investigación académica y comercial? y ¿qué líneas de investigación pueden derivarse? El artículo expone las principales oportunidades y retos que enfrenta esta disciplina en el ámbito académico.

Palabras clave: neurociencias del consumidor, Consumer neuroscience: 


\title{
CONSUMER NEUROSCIENCE: AN ACADEMIC VISION OF ITS RECENT EVOLUTION
}

Article for review

\begin{abstract}
This article analyzes the recent evolution in the theoretical and practical applications of consumer neuroscience. Identifying some of the milestones of this discipline, such as the adoption of the term "neuro-marketing», the early publications in academic journals, and the growing proliferation of neuroscience to solve marketing problems both at commercial and academic levels, this paper tries to answer the following questions: how has neuroscience evolved and impacted marketing? What are some of the main tools neuroscience offers in the field of academic and commercial research? What investigation lines could result from it? This article describes some of the opportunities and challenges facing this discipline in the academic world.
\end{abstract}

Key words: consumer neuroscience, neuro-marketing, behavior, consumer 


\section{Introducción}

En 2002, se acuña por primera vez el término «neuromarketing», por parte del profesor Ale Smidts, quien fue el primero en nombrar el uso de técnicas neurocientíficas en la disciplina del marketing (Solnais et al., 2013). En el ámbito académico, aparecen publicaciones con el mismo término a mediados de 2007 (Lee et al., 2018); a partir de allí, el tema ha tenido gran aceptación tanto en el ámbito comercial, para proveer soluciones a problemas empresariales, como académico, donde las líneas de investigación que relacionan las neurociencias y el marketing han tenido creciente interés (Ramsøy, 2019).

A pesar de que los términos «neuromarketing» y «neurociencias del consumidor» pueden utilizarse como sinónimos, algunos autores hacen una distinción (Hubert \& Kenning, 2008; Solnais et al., 2013), debido a que «neuromarketing» puede referirse a la implementación práctica de los conocimientos generados por las neurociencias del consumidor (Hubert \& Kenning, 2008; Hubert, 2010). Este artículo utiliza preferentemente «neurociencias del consumidor», definido como «el estudio de las condiciones neurales y procesos que subyacen el consumo, su significado psicológico y consecuencias de comportamiento» (Reimann et al., 2011, p. 611); además de ser un término preferente en la literatura académica (Karmarkar y Yoon, 2016; Karmarkar y Plassman, 2019; Ramsøy, 2019).

El creciente interés por la disciplina es notable, según lo indican Lee et al. (2018) en años recientes. Revistas académicas importantes como Journal of Marketing Research en 2015, Association for Consumer Research y European Journal of Marketing, en 2016, destinaban números especiales para investigaciones relacionadas con neurociencias y marketing. Este auge de la investigación también ha arrojado alertas en relación a la interpretación y uso de las tecnologías por parte de investigadores de marketing, especialmente por la sofisticación del análisis que algunas herramientas requieren (Ruff \& Huettel, 2014; Baron et al., 2017).

El objetivo del presente artículo es mostrar una visión académica de las neurociencias del consumidor, para responder las siguientes preguntas: ¿cuál ha sido la evolución y el impacto de las neurociencias en el marketing?, ¿cuáles son los principales instrumentos que pueden utilizarse para la investigación académica y comercial? y ¿qué líneas de investigación pueden derivar para esta disciplina?

El resto del artículo se estructura de la siguiente forma: primero, se introduce la reciente evolución del término en la literatura académica. En el segundo apartado se describen los principales instrumentos de medición que se han utilizado en investigación práctica y neurocientífica del consumidor. Tercero, se presenta una discusión sobre las posibles líneas de investigación académica que pueden surgir con base en el estudio de la disciplina, para finalizar con la conclusión del trabajo. 


\section{Neurociencias del consumidor}

La década de los noventa fue declarada por el presidente de EE. UU. George W. Bush como «la década del cerebro», para promover la investigación científica del cerebro. A esta iniciativa se sumaron esfuerzos internacionales que promovieron avances significativos en la comprensión de uno de los órganos más importantes del ser humano, pero del que aún falta mucho por conocer. Como se mencionó en la introducción, en la siguiente década, del 2000-2010, el estudio del cerebro empieza a permear en la disciplina del marketing, al acuñar el término «neuromarketing» y dar a luz investigaciones sobre el tema, publicadas en revistas científicas (Lee et al., 2018). En esa misma década, también comienzan a surgir las primeras consultoras sobre el tema, y la disciplina se expande significativamente (Plassman et al., 2012). Con el fin de promover el neuromarketing a nivel internacional, en 2012 se establece la Neuromarketing Science and Business Association (NMSBA), que agrupa a los profesionales interesados en el ámbito. A la vez, diversas revistas científicas y conferencias académicas empiezan a promover la investigación en el tema (Solnais et al., 2013; Lee et al., 2017).

Uno de los estudios fundamentales para el desarrollo del neuromarketing fue el de McClure et al. (2004), quienes al utilizar la resonancia magnética funcional por imágenes (fMRI, por sus siglas en inglés), midieron la preferencia de los consumidores entre Pepsi y Coca-Cola, mostrando evidencia de la actividad neuronal en áreas específicas de la memoria (el hipocampo y la corteza prefrontal dorsolateral) cuando se veía la marca de Coca-Cola, pero no para Pepsi. Otro estudio clásico fue el realizado por Plassman et al. (2008), en el cual observaron cómo el cerebro reacciona al precio de los productos; en este caso dieron a los participantes el mismo vino, pero les indicaban que estaban probando dos vinos distintos con una diferencia considerable de precio (los escenarios variaban entre $\$ 5$ a \$90), concluyendo que las zonas cerebrales que experimentan placer (córtex medial orbifrontal), estaban más activas cuando el participante bebía el vino que parecía más costoso.

Los estudios han explorado diversos ámbitos del marketing como influencia del precio en la experiencia (Schmidt et al., 2017), desarrollo de modelos para ver los efectos de la publicidad (Reynolds \& Phillips, 2018), o conocer la disponibilidad a pagar por los productos (Ramsøy et al., 2018). Los elementos de marketing convencional, como el marketing mix, también han sido sujeto de estudio (Smidts et al., 2014; Solnais et al., 2013).

La evolución e integración de las neurociencias con el marketing también ha reflejado, en algunos casos, conclusiones apresuradas. Sirva de ejemplo el caso 
de Martin Lindstrom, autor del best-seller Compradicción, cuando publica en el New York Times un artículo titulado "You Love Your iPhone. Literally» («Amas a tu iPhone. Literalmente»), en el cual afirma haber utilizado el fMRI para analizar lo que pasaba en el cerebro de los participantes, cuando timbraba su teléfono iPhone. Lindstrom indicaba que, al realizar esa acción, se activaba la corteza insular, la cual está relacionada con el amor, por lo tanto, los participantes «amaban a su iPhone. Literalmente» (Lindstrom, 2011). A los cuatro días, el New York Times publica un artículo firmado por cuarenta y cuatro neurocientíficos de prestigiosas universidades ${ }^{1}$, en donde indican que es necesario ser cautelosos en las conclusiones de los estudios del cerebro. En relación a este caso, anotaban que la corteza insular está activa en uno de cada tres estudios del cerebro y que, en estudios de toma de decisiones, dicha área está más asociada con emociones negativas que positivas (Poldrack, 2011).

Existen varios términos que la literatura académica ha dado a este tipo de malinterpretaciones. Por ejemplo, «pornografía cerebral» (Waytz \& Mason, 2013), para referirse a las simplificaciones o interpretaciones erróneas que se han dado a los elementos científicos de las neurociencias, o bien «correlaciones vudú» (Plassmann et al., 2015; Baron et al., 2017) para hacer referencia al problema de la inferencia inversa, que conlleva a conclusiones erróneas, confundiendo «correlación con causalidad». Para demostrar la magnitud de esta situación, Bennett et al. (2010) publican un artículo utilizando el fMRI con un salmón muerto para demostrar la magnitud y el problema de los falsos positivos en este tipo de estudios.

${ }_{1}^{1}$ La lista de los firmantes puede consultarse en: http://www.russpoldrack.org/2011/10/nyt-letter-toeditor-uncut-version.html 


\section{Mediciones en neuromarketing ${ }^{2}$}

Las tecnologías de medición en neuromarketing, pueden dividirse en dos apartados: medición de cambios en el cerebro y cambios en el cuerpo. Las herramientas que ayudan a medir procesos cerebrales han sido desarrolladas recientemente. Las dedicadas a medir cambios del cuerpo, datan de los años setenta, teniendo sus inicios en la psicología. Sin embargo, han evolucionado a través de los años, siendo ahora más precisas y portátiles (Schwarzkopf, 2015).

La tabla 1 resume las principales características de las herramientas más utilizadas. Para una clasificación con otros detalles, se recomienda ver Harrell (2019), para una selección de compañías de neuromarketing y los productos que ofrecen.

\section{Tabla 1. Tecnologías de neuromarketing}

\begin{tabular}{|l|l|}
\multicolumn{1}{|c|}{$\begin{array}{c}\text { Herramientas de } \\
\text { neuro-imágenes }\end{array}$} & \multicolumn{1}{c|}{ Medición de: } \\
\hline $\begin{array}{l}\text { Resonancia magnética funcional } \\
\text { por imágenes (fMRI) }\end{array}$ & $\begin{array}{l}\text { Cambio en el nivel de oxigenación del flujo } \\
\text { sanguíneo en el cerebro. }\end{array}$ \\
\hline Electroencefalograma (EEG) & $\begin{array}{l}\text { Cambios en la actividad eléctrica del cerebro } \\
\text { en el cuero cabelludo. }\end{array}$ \\
\hline Magnetoencefalografía (MEG) & Cambio en los campos magnéticos. \\
\hline Herramientas fisiológicas & $\begin{array}{l}\text { Seguimiento del movimiento y fijación de } \\
\text { la pupila. }\end{array}$ \\
\hline Eye-tracking & $\begin{array}{l}\text { Reacción de músculos faciales ante las } \\
\text { emociones generadas. }\end{array}$ \\
\hline Decodificación facial & $\begin{array}{l}\text { Cambios en el nivel de sudor en la palma de las } \\
\text { manos o pies. }\end{array}$ \\
\hline Respuesta galvánica & Cambios en la actividad eléctrica del corazón. \\
\hline Electrocardiograma &
\end{tabular}

Fuente: adaptado de Dimoka et al. (2012).

\footnotetext{
${ }^{2}$ Este apartado recoge algunos elementos del capítulo 6 de la obra de Paredes (en prensa).
} 
Plassman y Karmarkar (2015) presentan los resultados de una encuesta realizada a académicos y profesionales que utilizaban las neurociencias para investigar problemas de marketing. Los resultados demuestran que, para los académicos, el fMRI era la herramienta más utilizada, seguida por el eye-tracker. Para los profesionales, el eye-tracker es el de mayor uso, seguido por el EEG. De acuerdo a los autores, las diferencias pueden darse por el costo de las distintas herramientas. Los resultados en el ámbito académico son consistentes con la revisión de literatura realizada por Lee et al. (2018), en donde desde los años 2007-2016 la tecnología más utilizada es el fMRI, seguido por el EEG, cuyo uso reciente ha incrementado del 2014 al 2016.

Tanto el fMRI como el EEG datan desde la década de los noventa (Ruanguttamanun, 2014), y con el tiempo han ido perfeccionándose. Cuando existe actividad neuronal en un área del cerebro, esta consume mayor cantidad de oxígeno: el fMRI mide este incremento del nivel de oxigenación en el flujo sanguíneo, permitiendo ver las áreas específicas que reaccionan ante un estímulo; esto se conoce como señales BOLD por sus siglas en inglés (blood oxygenation level dependent). EI EEG, similar a una gorra de tela con electrodos, mide esta actividad a través de sensores eléctricos que amplifican estas señales para establecer las reacciones de los consumidores cuando son expuestos a un estímulo. Finalmente, el eye-tracker consiste en una cámara con rayos infrarrojos que siguen el centro de la pupila del ojo, donde la luz es reflejada desde la córnea, lo que permite contar con una serie de métricas muy útiles para la toma de decisiones.

En relación al tamaño de las muestras, el número sigue siendo tema de debate. Debido al costo de la tecnología, los estudios de neurociencias del consumidor emplean números relativamente pequeños, en comparación con los estudios tradicionales de marketing. Mas allá del número de participantes en este tipo de estudios, también intervienen otros elementos como invasión, precisión estimada, replicabilidad, entre otros (Butler et al., 2017; Friston, 2012). Como referencia, Ramsøy (2019) indica que el tamaño mínimo recomendable es de treinta participantes; sin embargo, como se ha mencionado, esto dependerá de las características particulares de cada estudio.

A nivel latinoamericano, aún faltan esfuerzos significativos para el desarrollo de la disciplina. En el ámbito comercial han surgido firmas locales y representaciones de consultoras internacionales que operan en distintos países. En el ámbito académico, aún continúa siendo una asignatura pendiente: con algunas excepciones (Avendaño et al., 2015; Juárez et al., 2019) de publicaciones sobre la materia, no es usual encontrar investigación empírica en neurociencias del consumidor, pues el enfoque ha sido más divulgativo (Braidot, 2011). 


\section{Investigación científica en neurociencias del consumidor}

La investigación científica en la disciplina ha ido en crecimiento. En la revisión de 131 artículos académicos, publicados entre 2007 y 2016, Lee et al. (2018) identifican las tendencias de investigación, además de las tecnologías más utilizadas mencionadas en la sección anterior. El estudio demuestra que la cantidad de trabajos conceptuales (85) aún sobrepasa los trabajos empíricos (45), sin embargo, los trabajos empíricos han sido publicados en journals de mayor impacto. Los autores recomiendan realizar investigación integrando otras tecnologías, que complementen el protagonismo del fMIR y EEG, así como pasar de la investigación centrada en el cerebro como sistema modular reactivo, a una red dinámica de la actividad cerebral, es decir, respuestas más espontáneas, como funciona en realidad.

En los últimos años, se han hecho avances significativos para entender cómo las neurociencias pueden incidir en la toma de decisiones del consumidor. Las nuevas fronteras podrían estar definidas por la inclusión de la genética y neurociencia molecular, y la injerencia de los métodos computacionales para mejorar las tecnologías para la generalización de los resultados del cerebro (Smidts et al., 2014).

Otros autores, dividen e identifican diversos ámbitos de investigación en neurociencias del consumidor. Por ejemplo, Lee et al. (2007) establecen algunas áreas de estudio como:

1. Confianza: ¿se activan las mismas zonas cerebrales cuando se confía en lo que dice un vendedor, un comercial o un amigo? ¿Cómo se procesa la información cuando se confía en un comercial o cuando es producto de la referencia de un amigo? ¿De qué forma evoluciona la confianza producto de la relación con la empresa o lealtad con la marca?

2. Precio: ¿los individuos realmente ignoran los dos dígitos finales cuando están procesando la información? ¿Es el precio producto de la información racional, o está relacionado con activaciones de zonas emocionales? ¿Qué efectos tiene la marca en el precio?

3. Ética: ¿por qué algunos individuos se convierten en usuarios de crédito compulsivo? ¿Qué zonas cerebrales se activan cuando se compra o se ve un mensaje de este tipo, versus quienes mantienen un nivel más apropiado de gasto? En el ámbito de ventas, ¿se puede diferenciar la actividad del cerebro de los vendedores que aplican principios éticos, y aquellos que realizan acciones menos éticas? ¿Son los individuos menos éticos más dispuestos a fijar objetivos a corto plazo para obtener beneficios inmediatos? 
Solnais et al. (2013) dividen las áreas de investigación en cuatro grandes pilares:

1. Toma de decisiones: ¿cómo se forman las preferencias del consumidor y cómo puede predecirse la actividad neuronal?

2. Recompensas: ¿cómo los estímulos de marketing activan el sistema de recompensa del cerebro e influencian el valor percibido?

3. Memoria: ¿cómo se forma la memoria en relación a los productos y la publicidad, y cómo influencia el comportamiento del consumidor?

4. Emociones: ¿cómo son procesados los estímulos en una base motivacional y emocional?

A pesar de los esfuerzos en el área, sin duda son muchas preguntas que aún faltan por responder para tener una mejor comprensión del consumidor. 


\section{Conclusión}

El artículo presentó una perspectiva académica de las neurociencias del consumidor, mostrando su reciente evolución y las principales herramientas de investigación para discutir algunas líneas futuras de investigación. Después de más de tres décadas desde que empiezan a surgir elementos de neuromarketing, se pueden develar grandes avances sobre cómo funciona el cerebro en los procesos de toma de decisiones; sin embargo, aún queda mucho por descubrir para seguir avanzando. Las herramientas, de las que algunas cuentan con más de tres décadas, han mejorado su tecnología para mejorar la precisión y ser menos invasivas. Finalmente, aún se requiere más investigaciones para mejorar la comprensión y el desarrollo en el conocimiento del consumidor desde las neurociencias. 


\section{Referencias}

Avendaño, W. R., Paz, L. S. y Rueda, G. (enero-junio 2015). Estímulos auditivos en prácticas de neuromarketing. Caso: Centro Comercial Unicentro, Cúcuta, Colombia. Cuadernos de Administración, 31(53), 117-129.

Baron, A. S., Zaltman, G. \& Olson, J. (2017). Barriers to advancing the science and practice of marketing. Journal of Marketing Management, 33(11/12), 893-908.

Bennett, C. M., Baird, A. A., Miller, M. B. \& Wolford, G. L. (2010). Neural correlates of interspecies perspective taking in the Post-Mortem Atlantic Salmon: An Argument for Proper Multiple Comparisons Correction. Journal of Serendipitous and unexpected results, 1(1), 1-5.

Braidot, N. (2011). Neuromarketing en acción. ¿Por qué tus clientes se acuestan con otros si dicen que les gustas tú? Argentina: Granica.

Butler, M. J., Lee, N. \& Senior, C. (2017). Critical Essay: Organizational Cognitive Neuroscience Drives Theoretical Progress, or: The Curious Case of the Straw Man Murder. Human Relations, 70(10), 1171-1190.

Dimoka, A., Banker, R. D., Benbasat, I. Davis, F. D., Dennis, A. R. \& Gefen, D. (2012). On the use of neurophysiological tools in information systems research: developing a research agenda for NeuroIS. MIS Quarterly, 36(3), 679-702.

Friston, K. (2012). Ten Ironic Rules for Non-Statistical Reviewers. Neuroimage, $61(4), 1300-1310$.

Glaenzer, E. (2016). Are the Brain and the Mind One? Neuromarketing and How Consumers Make Decisions. Honors Theses. Paper 812.

Harrell, E. (enero 2019). Neuromarketing. What you need to know. Harvard Business Review, 2-8.

Hsu, M. (2017). Neuromarketing: Inside the Mind of the Consumer. California Management Review, 59(4), 5-22.

Hubert, M. \& Kenning, P. (2008). A current overview of consumer neuroscience. Journal of Consumer Behaviour, 7(4-5), 272-292.

Hubert, M. (2010). Does neuroeconomics give new impetus to economic and consumer research? Journal of Economic Psychology, 31(5), 812-817.

iMotions. (2017). Eye-Tracking. The Complete Pocket Guide. 
Juárez, D., Tur-Viñes, V. y Mengual, A. (2019). Análisis del diseño de packaging de juguete educativo, mediante neuromarketing. Cuadernos Latinoamericanos de Administración, XVI(28).

Karmarkar, U. \& Plassman, H. (2019). Consumer Neuroscience: Past, Present, and Future. Organizational Research Methods, 22(1), 174-195.

Karmarkar, U. R. \& Yoon, C. (2016). Consumer neuroscience: Advances in understanding consumer psychology. Current Opinion in Psychology, 10, 160165. doi: https://doi.org/10.1016/j.copsyc.2016.01.010

Lee, N., Brandes, L., Chamberlain, L. \& Senior, C. (2017). This is your brain on neuromarketing: Reflections on a decade of research. Journal of Marketing Management, 33(11-12), 878-892.

Lee, N., Broderick, A. \& Chamberlain, L. (2007). What is 'neuromarketing'? A discussion and agenda for future research. International Journal of Psychophysiology. 63(2), 199-204.

Lee, N., Chamberlain, L. \& Brandes, L. (2018). Welcome to the jungle! The neuromarketing literature through the eyes of a newcomer. European Journal of Marketing, 52(1/2), 4-38. doi: 10.1108/EJM-02-2017-0122.

Lindstrom, M. (2011). You Love Your iPhone. Literally. The New York Times. Recuperado de https://www.nytimes.com/2011/10/01/opinion/you-love-youriphone-literally.html

McClure, S. M., Li, J., Tomlin, D., Cypert, K. S., Montague, L. M. \& Montague, P. R. (2004). Neural correlates of behavioral preferences for culturally familiar drinks. Neuron, 44, 379-387.

Ohme, R., Reykowska, D., Wiener, D. \& Choromanska, A. (2009). Analysis of neurophysiological reactions to advertising stimuli by means of EEG and Galvanic skin response measures. Journal of Neuroscience, Psychology, and Economics, 2(1), 21-31.

Paredes, M. R. (2020). Consumerología. Bogotá, Colombia: LID Editorial.

Plassmann, H. \& Karmarkar, U. R. (2015). Consumer neuroscience: Revealing meaningful relationships between brain and consumer behavior. En C. Lamberton, M. Norton \& D. Rucker (Eds.), Cambridge handbook of consumer psychology (pp. 23-35). Cambridge: Cambridge University Press.

Plassmann, H., Ramsøy, T. Z. \& Milosavljevic, M. (2012). Branding the Brain: A Critical Review and Outlook. Journal of Consumer Psychology, 22(1), 18-36.

Poldrack, R. (2011). The iPhone and the Brain. The New York Times. Recuperado de https://www.nytimes.com/2011/10/05/opinion/the-iphone-and-the-brain.html 
Puškarević, I., Nedeljković, U., Dimovski, V. \& Možina, K. (2016). An eye tracking study of attention to print advertisements: Effects of typeface figuration. Journal of Eye Movement Research, 9(5), 1-18.

Ramsøy, T. Z. (2019). A foundation for consumer neuroscience and neuromarketing. Journal of Advertising Research [working paper], 1-32.

Ramsøy, T. Z., Skov, M., Christensen, M. K. \& Stahlhut, C. (2018). Frontal brain asymmetry and willingness to pay. Frontiers in Neuroscience, 12(3), 13-24.

Reimann, M., Schilke, O., Weber, B., Neuhaus, C. \& Zaichkowsky, J. (2011). Functional magnetic resonance imaging in consumer research: $A$ review and application. Psychology and Marketing, 28(6), 608-637.

Reynolds, T. J. \& Phillips, J. M. (2018). The Strata Model Predicting Advertising Effectiveness: A Neural-Network Approach Enhances Predictability of Consumer Decision Making. Journal of Advertising Research, 59(3), 268-280.

Ruanguttamanun, C. (2014). Neuromarketing: I put myself into a fMRI scanner and realized that I love Louis Vuitton ads. Procedia - Social and Behavioral Sciences, 148, 211-218.

Ruff, C. C. \& Huettel, S. A. (2014). Experimental methods in cognitive neuroscience.

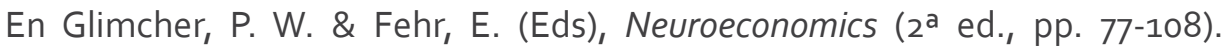
Amsterdam: Academic Press.

Schmidt, L., Skvortsova, V., Kullen, C., Weber, B. \& Plassmann, H. (2017). How context alters value: Price information recruits the brain's valuation and affective regulation system for shaping experienced taste pleasantness. Scientific Reports, 7(8), 1-13.

Schwarzkopf, S. (2015). Measurement devices and the psychophysiology of consumer behavior: A posthuman genealogy of neuromarketing. BioSocieties, 10(1), 1-18.

Smidts, A., Hsu, M., Sanfey, A. G., Boksem, M. A. S., Ebstein, R. B., Huettel, S. A., Kable, J. W., Karmarkar, U. R., Kitayama, S., Knutson, B., Liberzon, I., Lohrenz, T., Stallen, M. \&Yoon, C. (2014). Advancing consumer neuroscience. Marketing Letters, 25(3), 257-267

Solnais, C., Andreu-Perez, J., Sánchez-Fernández, J. \& Andréu-Abela, J. (2013). The contribution of neuroscience to consumer research: A conceptual framework and empirical review. Journal of Economic Psychology, 36(2), 68-81. doi: http:// dx.doi.org/10.1016/j.joep.2013.02.011

Waytz, A. \& Mason, M. (julio-agosto 2013). Your Brain at Work: What a new approach to neuroscience can teach us about management. Harvard Business Review, 1-9. 